\title{
Very High Sustainable Forward Current Densities on 4H-SiC p-n Junctions formed by VLS localized epitaxy of heavily Al-doped $p^{++}$emitters
}

\author{
S. Sejil ${ }^{1,2 a^{*}}$, L. Lalouat ${ }^{1,2 b}, M$. Lazar $^{2 c}$, D. Carole $^{1 \mathrm{~d}}$, C. Brylinski ${ }^{1 \mathrm{e}}$, \\ F. Jomard ${ }^{3 f}$, D. Planson ${ }^{2 g}$, G. Ferro ${ }^{\text {hh }}$, C. Raynaud ${ }^{2 \mathrm{i}}$, \\ ${ }^{1}$ Laboratoire des Multimatériaux et Interfaces, UMR 5615, Université de Lyon 1, CNRS, \\ 69622 Villeurbanne, France \\ ${ }^{2}$ AMPERE, INSA Lyon, Ecole Centrale de Lyon, CNRS, 69621 Villeurbanne, France \\ ${ }^{3}$ GEMAC, Université Versailles-Saint-Quentin, CNRS, 78035 Versailles, France

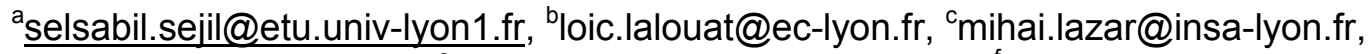 \\ ddavy.carole@univ-lyon1.fr, ${ }^{\circ}$ christian.brylinski@univ-lyon1.fr, ${ }^{\prime}$ francois.jomard@uvsq.fr

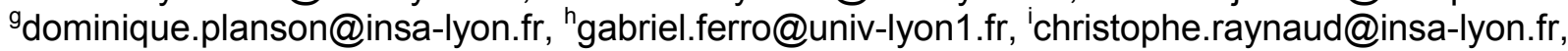

Keywords: SiC, $\mathrm{p}^{++}$doping, VLS, selective epitaxy, roughness, p-n Junction, p-type ohmic contact.

\begin{abstract}
This study deals with the electrical characterization of $\mathrm{PiN}$ diodes fabricated on a $4^{\circ}$ off-axis $4 \mathrm{H}-\mathrm{SiC} \mathrm{n}^{+}$substrate with a $\mathrm{n}^{-}$epilayer $\left(1 \times 10^{16} \mathrm{~cm}^{-3} / 10 \mu \mathrm{m}\right)$. Optimized $\mathrm{p}^{++}$epitaxial areas were grown by Vapour-Liquid-Solid (VLS) transport to form $\mathrm{p}^{+}$emitters localized in etched wells with $1 \mu \mathrm{m}$ depth. Incorporated Al level in the VLS p ${ }^{++}$zones was checked by SIMS (Secondary Ion Mass Spectroscopy), and the doping level was found in the range of $1-3 \times 10^{20}$ at. $\mathrm{cm}^{-3}$. Electrical characterizations were performed on these PiN diodes, with $800 \mathrm{~nm}$ deposit of aluminium as ohmic contact on p-type SiC. Electrical measurements show a bipolar behaviour, and very high sustainable forward current densities $\geq 3 \mathrm{kA} \cdot \mathrm{cm}^{-2}$, preserving a low leakage current density in reverse bias. These measurements were obtained on structures without any passivation and no edge termination.
\end{abstract}

\section{Introduction}

Continuous improvement of bulk and epilayers of $4 \mathrm{H}-\mathrm{SiC}$ semiconductor materials, allows currently the marketing of power devices. However, technological difficulties still remain unresolved, like the implementation of a fully satisfactory process to locate p-type doping area into 4H-SiC. Indeed, ion implantation is the only viable technique in industry to perform localized $\mathrm{p}^{+}$doping, however with significant drawbacks [1]. The damages created during ion implantation require a high temperature annealing $\left(\sim 1700^{\circ} \mathrm{C}\right.$ or higher [2]) to both partially activate the $\mathrm{Al}$ elements and restore the crystal quality. Moreover, in practice, with standard ion implanter energy $(<200 \mathrm{keV})$, the depth of the p-type implanted areas is limited to a few hundreds of nm, which can lead in turn to severe design restrictions. To solve this problem, VLS localized epitaxy has been previously proposed by some of the authors as an alternative to implement deeper localized Al doping at high concentrations [3, 4]. Further works have resulted in the improvement of localized VLS process, yielding optimized structural morphology [5], preserving a threshold voltage of $\sim 3 \mathrm{~V}$ in forward bias, value predicted by the classical theory, on a $4 \mathrm{H}-\mathrm{SiC}$ p-n junction, and eliminating the need of high temperature post-growth annealing (previously $\sim 1700^{\circ} \mathrm{C}$ ) [6]. To the best of our knowledge, this electrical behaviour has never been obtained before with such low temperature doping process $\left(1100^{\circ} \mathrm{C}\right)$. This study was followed with a new one whose aim was to increase the deposit thickness up to $1 \mu \mathrm{m}$ [7]. Implementing these new p-type VLS layers with an adequate technological process has allowed us to obtain $4 \mathrm{H}-\mathrm{SiC}$ diodes withstanding spectacular high current densities in forward bias, and providing low leakage current densities in reverse bias. Hereafter are presented elements of the technological process and the J-V characteristics of these diodes which clearly present a bipolar behavior (light emission in forward bias). 


\section{Experimental section}

The manufacturing process to prepare the sample, and details about the optimization of VLS localized epitaxy have been reported elsewhere [7]. After VLS growth sequence, the amount of Al incorporated into the grown layer (doping profile) was determined by Secondary Ion Mass Spectrometry (SIMS) for different diameters of the wells. The doping uniformity was checked.

Then, to fabricate the PiN diodes (see Fig.1), classical Ni-based ohmic contacts were deposited on the back side of the n-type substrate by e-beam evaporation, followed by a Rapid Thermal Annealing (RTA) at $900^{\circ} \mathrm{C}$ in argon atmosphere. Because of the high doping level of the VLS $\mathrm{p}^{++}$epilayers, it was possible to get ohmic contacts on these p-type layers just by depositing an Al layer (by sputtering), i.e. without annealing this metallization. This Al layer was thick enough $(800 \mathrm{~nm})$ to get full coverage of the rather rough VLS epilayers surface (see Fig. 2), presenting a pronounced step-bunching which is a good evidence that the growth from the melt occurred in a homo-epitaxial way.

The as-fabricated PiN diodes were electrically characterized in order to validate the quality of the pn junction, using a Keithley 2636 System Measure Unit with a "Signatone S1 160" probe station. These measurements were done on vertical diode configuration, at room temperature. Note that neither passivation nor edge termination (such as guard rings, mesa or JTE) were used.

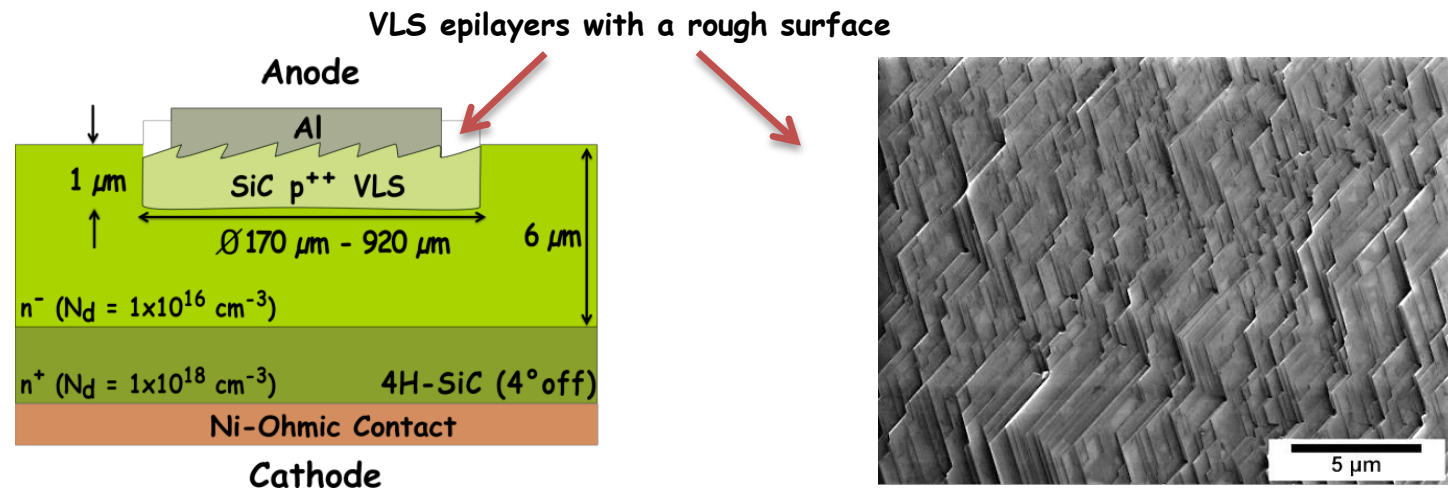

Figure 1: Schematic cross sectional view of the fabricated PiN diodes, with $\mathrm{p}^{++}$emitter formed by VLS epitaxy

Figure 2 : SEM image (top view), surface morphology over a VLS thick layer

\section{Results and discussions}

As a first result, most of the aluminum concentration values measured by SIMS were in the range $1-3 \times 10^{20}$ at.cm ${ }^{-3}$ (see Fig. 3) with a nearly constant value over the layer thickness, clearly visible for the largest diodes ( $\varnothing 920$ $\mu \mathrm{m})$ where the deposit thickness is $\sim 900 \mathrm{~nm}$. It should be emphasized that such a high and constant doping level is quite difficult to be reached by standard CVD or by ion implantation without degradation of the layer crystalline quality. The thickness values, measured by SIMS, in the range of 200-900 $\mathrm{nm}$, are in agreement with those measured by Tencor stylus profilometer from the difference between the depth of the etched well, before and after VLS growth [7].

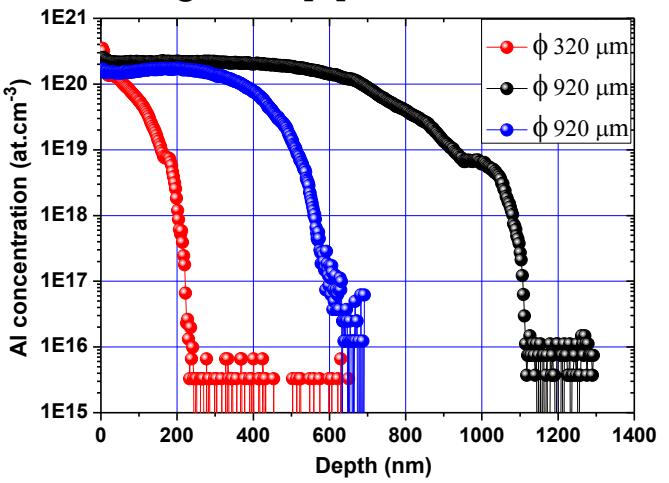

Figure 3: SIMS depth profiles of Al concentration in SiC layers grown by VLS 
Typical current density vs. voltage (J-V) characteristics, measured on diode structures with VLS grown emitters are reported in Fig. 4. For a better readability, only a few J-V characteristics amongst the more representative have been plotted. The total current is limited to $1 \mathrm{~A}$ in all cases due to instrumental limitations of the K2636 SMU unit.
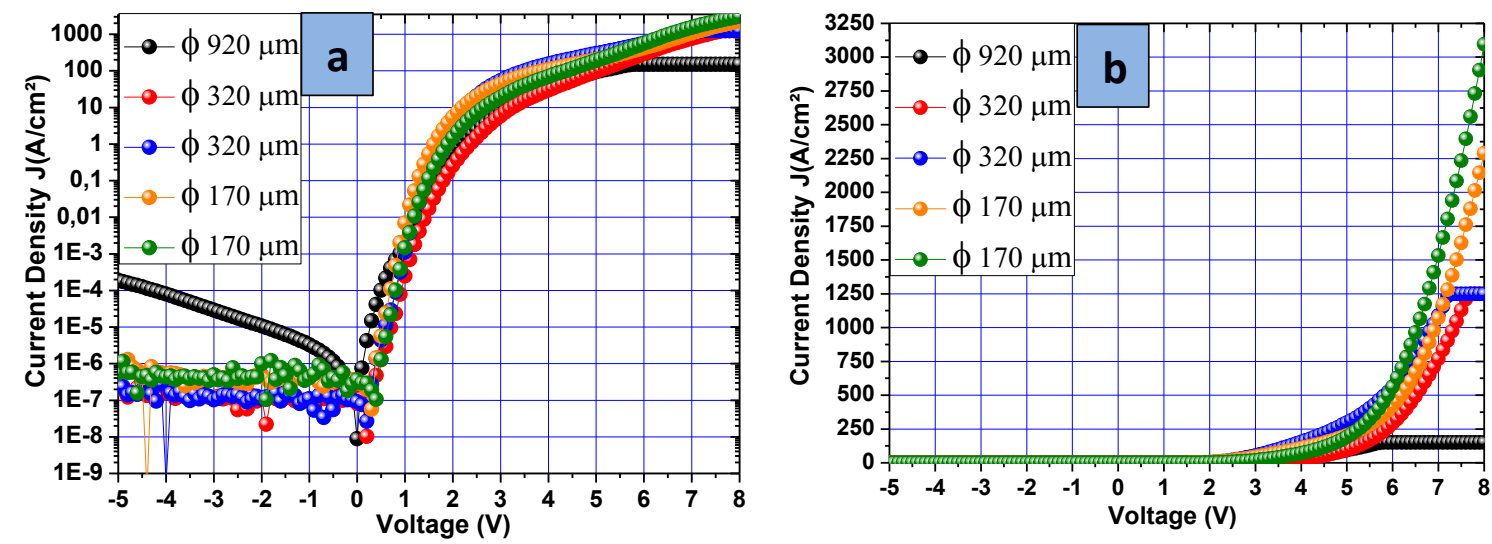

Figure 4: J-V behaviors as a function of the diameter of the well

(a) Reverse and forward bias in logarithmic scale and (b) forward bias in linear scale.

In forward bias, PiN diodes exhibit spectacular high and sustainable current densities: from 150

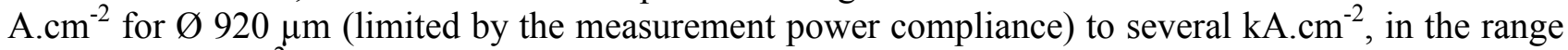
1250-3000 A.cm ${ }^{-2}$ for the smaller ones. Measured threshold voltages are around $\sim 3 \mathrm{~V}$, which is the typical threshold voltage for $4 \mathrm{H}-\mathrm{SiC}$ p-n junction. Moreover, as an improvement over a previous report [7], the current density curves overlap quite well in forward bias, confirming that the metal deposit of the front side top contact ( $800 \mathrm{~nm}$ thickness) is now thick enough to fully cover the whole VLS layers. Such an optimized front side top contact avoids high electric field spots inducing premature breakdown. In reverse bias, leakage current density is rather low, measured in the range of $10^{-6}-10^{-7}$ A.cm ${ }^{-2}$ for diodes of smaller diameters, and surprisingly higher for $\varnothing 920 \mu \mathrm{m}$, measured in the range of $10^{-5}-10^{-4} \mathrm{~A} . \mathrm{cm}^{-2}$, unlike previously reported value of leakage current density of $\sim 10^{-8} \mathrm{~A} . \mathrm{cm}^{-2}$ [7]. The high leakage current density for $\varnothing 920 \mu \mathrm{m}$ diodes is probably due to device processing issues. On the border of the anode contact, the metal is probably not completely etched and it slightly overlaps with the n-zone at the well edge, resulting in a parasitic Schottky contact in parallel with the p-n junction.

Then, repetitive current-voltage measurements in reverse regime were carried out. A series of tests at the same reverse voltage was performed on the same diode in order to study the evolution of the leakage current. Compliance has been fixed at 10 or $100 \mu \mathrm{A}$, depending on the test, in order to avoid the destruction of the diode. Fig. 5 reports results of measurements performed on $\varnothing 170 \mu \mathrm{m}$ diodes.

In order to compare our results with those previously presented in [6], breakdown voltage tests were performed under the same conditions, i.e. in air and at room temperature. In the present case, breakdown voltage $\mathrm{V}_{\mathrm{BR}}$ is estimated at $-420 \mathrm{~V}$. Due to the low compliance at $10 \mu \mathrm{A}$, the diode is not destroyed when breakdown begins at $\sim 420 \mathrm{~V}$. In the second test, with the same compliance, prebreakdown leakage current is a little higher. No degradation is observed between $2^{\text {nd }}$ and $3^{\text {rd }}$ test. But for the third test, compliance has been set to $100 \mu \mathrm{A}$, and subsequently the diode has been a little more degraded. 


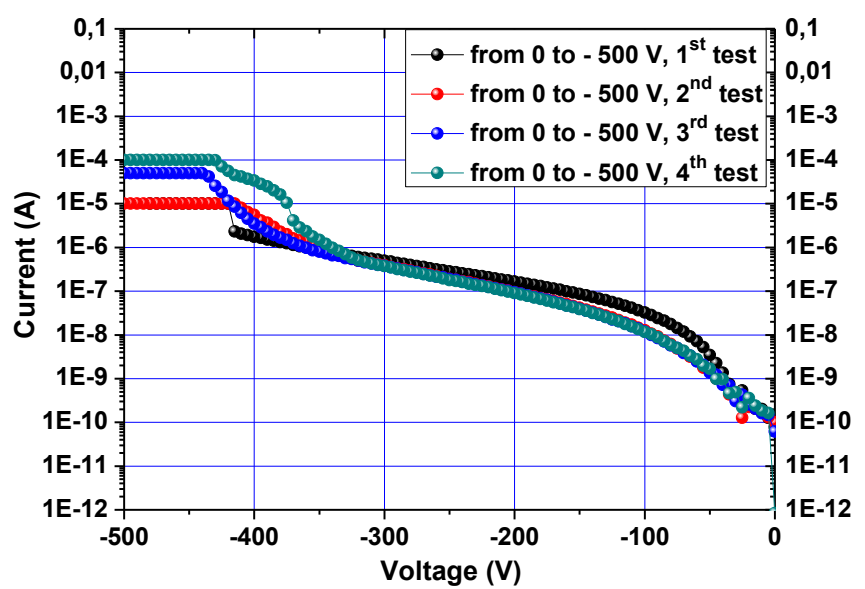

Figure 5: Breakdown voltage test of PiN diodes (Ø $170 \mu \mathrm{m})$ without passivation or edge termination
Indeed, on the $4^{\text {th }}$ pre-breakdown test, the current increases drastically at $\sim 370 \mathrm{~V}$. For such a doping level and thickness, $420 \mathrm{~V}$ represents $\eta=38 \%$ of the theoretical $V_{B R}$ calculated in ideal 1D planar model. $\eta$ is the "blocking efficiency" of the junction. In a previous study [6], the experimental breakdown voltage was as low as $50 \mathrm{~V}$, which represented only $2 \%$ of the theoretical value. As a result, the net increase of the blocking efficiency confirms the beneficial effects of the crystalline quality optimization of the VLS epilayers.

\section{Conclusion}

SIMS analysis were performed on $\mathrm{p}^{++}$VLS epilayers, and a doping level in the range of $1-3 \times 10^{20}$ at. $\mathrm{cm}^{-3}$ has been measured, confirming deposit thickness in the range of 200-900 nm. Then, PiN structures were implemented including these optimized $\mathrm{p}^{++}$VLS layers, together with an optimized technological process, which has provided $4 \mathrm{H}-\mathrm{SiC}$ diodes presenting a bipolar behaviour, and very high sustainable forward current densities $\geq 3 \mathrm{kA} . \mathrm{cm}^{-2}$ while preserving a low leakage current density in reverse bias. These results were obtained without passivation and edge termination. Reverse I-V measurements were carried out and a net increase in blocking efficiency, up to $38 \%$, was found, as compared to $2 \%$ in our previous study before optimization of VLS growth process. These improvements in blocking efficiency confirm the beneficial effects of the VLS crystalline quality optimization [7]. Even better performances can be expected in the future from diodes with adequate passivation and edge termination.

Acknowledgements. The authors gratefully thank NanoLyon Technological Platform staff for process expertise. This work has been financially supported by ST Microelectronics Company. LMI and AMPERE labs are members of the I@L Carnot Institute.

\section{References}

[1] V. Heera, A. Mucklich, C. Dubois, M. Voelskow, W. Skorupa, J. of App. Phy., Vol. 96 (2004) p. 2841-2852. [2] M. Lazar, C. Raynaud, D. Planson, M.L. Locatelli, K. Isoird, L. Ottaviani, J.P. Chante, R. Nipoti, A. Poggi, G. Cardinali, Mater. Sci. Forum, Vols. 389-393 (2002) p. 827-830.

[3] D. Carole, S. Berckmans, A. Vo-Ha, M. Lazar, D. Tournier, P. Brosselard, V. Souliere, L. Auvray, G. Ferro, C. Brylinski, Silicon Carbide and Related Materials 2011, 717-720 (2012) 169-172.

[4] A. Vo-Ha, D. Carole, M. Lazar, D. Tournier, F. Cauwet, V. Soulière, N. Thierry-Jebali, P. Brosselard, D. Planson, C. Brylinski, G. Ferro, Thin Solid Films, Vol. 548 (2013) p. 125-129.

[5] S. Sejil, M. Lazar, F. Cayrel, D. Carole, C. Brylinski, D. Planson, G. Ferro, C. Raynaud, Mater. Sci. Forum, Vol. 858 (2016) p. 205-208.

[6] N. Thierry-Jebali, M. Lazar, A. Vo-Ha, D. Carole, V. Souliere, F. Laariedh, J. ul Hassan, A. Henry, E. Janzen, D. Planson, G. Ferro, C. Brylinski, P. Brosselard, Mater. Sci. Forum, Vols. 740-742 (2013) p. 911-914. [7] S. Sejil, M. Lazar, D. Carole, C. Brylinski, D. Planson, G. Ferro, C. Raynaud, Phys. Status Solidi A, DOI 10.1002/pssa.201600454 (2016). 